\title{
Angiogenesis in a rat model following myocardial infarction induced by hypoxic regulation of VEGF $_{165}$ gene-transfected EPCs
}

\author{
QIANG SHE ${ }^{1}$, SHUANG XIA $^{2}$, SONG-BAI DENG $^{1}$, JIAN-LIN DU ${ }^{1}$, \\ YE-QING $\mathrm{LI}^{3}$, LI HE ${ }^{1}$, JUN XIAO ${ }^{4}$ and YU-LUAN XIANG ${ }^{1}$
}

${ }^{1}$ Department of Cardiology, Second Affiliated Hospital, Chongqing Medical University; ${ }^{2}$ Department of Cardiology,
Geriatrics Institute, Guangdong General Hospital; ${ }^{3}$ Department of Cardiology, Third People's Hospital;
${ }^{4}$ Department of Cardiology, Emergency Centre Hospital, Chongqing 400010, P.R. China

Received May 13, 2012; Accepted September 12, 2012

DOI: $10.3892 / \mathrm{mmr} .2012 .1112$

\begin{abstract}
Hypoxia-response elements (HREs) regulate the expression of the vascular endothelial growth factor 165 $\left(\mathrm{VEGF}_{165}\right)$ gene and enhance the safety and efficacy of therapeutic angiogenesis. However, the role of hypoxic regulation of VEGF $_{165}$ gene-modified stem cells in promoting angiogenesis in the ischemic myocardium remains unclear. In this study, the effects of the hypoxic regulation of genetically modified endothelial progenitor cells (EPCs) on angiogenesis in the ischemic myocardium and on changes in cardiac function following acute myocardial infarction (AMI) were investigated through the transplantation of hypoxia-regulated $\mathrm{VEGF}_{165}$ gene-modified EPCs into the ischemic myocardium. Rat bone marrow-derived EPCs transfected with plasmid p6HRE-CMV-VEGF ${ }_{165}$ (6HRE-VEGF $\left.{ }_{165}-\mathrm{E}\right)$, and plasmid pCMV-VEGF ${ }_{165}\left(\mathrm{VEGF}_{165}-\mathrm{E}\right)$ were injected into rats with a successfully established model of AMI. The levels of $\mathrm{VEGF}_{165} \mathrm{mRNA}$ and protein expression in the EPCs and ischemic myocardium were determined using reverse transcription-polymerase chain reaction and western blot assay, respectively, and the capillary density in the ischemic myocardium and the cardiac function of the rats were detected using immunohistochemistry and echocardiography, respectively. We found that the hypoxia promoter 6HRE-CMV effectively regulated the expression of the $\mathrm{VEGF}_{165}$ gene in the EPCs and
\end{abstract}

Correspondence to: Professor Qiang She, Second Affiliated Hospital, Chongqing Medical University, 74 Linjiang Road, Yuzhong District, Chongqing 400010, P.R. China

E-mail: qshe98@hotmail.com

Abbreviations: HRE, hypoxic response element; EPCs, endothelial progenitor cells; AMI, acute myocardial infarction; $\mathrm{VEGF}_{165}$, vascular endothelial growth factor 165; Dil-ac-LDL, Dil-acetylated low density lipoproteins; RT-PCR, reverse transcription polymerase chain reaction; EF, left ventricular ejection fraction

Key words: angiogenesis, hypoxia, gene therapy, endothelial progenitor cells, myocardial infarction the ischemic myocardium. In rats of the $6 \mathrm{HRE}-\mathrm{VEGF}_{165}$-Etransplanted group, the levels of $\mathrm{VEGF}_{165}$ gene expression and capillary density in the ischemic myocardium were significantly higher than those in the other experimental groups. Moreover, cardiac function was also improved compared with that in other groups. VEGF 165 gene-modified EPCs are able to significantly promote angiogenesis in the ischemic myocardium and markedly ameliorate the cardiac function of rats following AMI, especially under 6HRE regulation.

\section{Introduction}

The vascular endothelial growth factor (VEGF) family is considered to be one of the most important pro-angiogenic factors during therapeutic angiogenesis. The VEGF family has been shown to not only reduce the infarction area, ameliorate cardiac function and increase angiogenesis in ischemic tissues during animal experiments (1-3), but also improve the ischemic symptoms of patients in partial phase I and II clinical trials $(4,5)$. However, there are some potential risks associated with the application of genes for therapeutic angiogenesis. For instance, overexpression and ectopic expression of genes may lead to angioma and promote carcinogenesis. Therefore, gene expression should be further regulated to enhance the safety and effectiveness of gene therapy.

Hypoxia response elements (HREs) are upstream enhancers of gene promoters under hypoxic regulation. They are able to induce the upregulation of target genes under hypoxic conditions. Multiple copies of HRE promoters have been found to increase the expression of target genes under hypoxic conditions, while the same genes were barely expressed in the presence of normal concentrations of oxygen, which increases the safety of gene therapy (6). Gene transport to target organs is important, but has been difficult to achieve to date. A number of studies have indicated that cultivated human endothelial progenitor cells (EPCs) or other sources of EPCs are able to reduce the area of myocardial infarction, providing a good strategy for treating patients with coronary atherosclerotic heart diseases, especially end-stage coronary heart diseases (7). Moreover, due to stem cell homing, the 
transportation of foreign genes into target organs may be realized, which improves the targeting effect of gene therapy. The VEGF gene is able to promote stem cell homing, stem cell differentiation and angiogenesis in ischemic tissues as well as improve the functions of ischemic tissues.

The effect of promoting angiogenesis using pro-angiogenic gene-modified stem cells under hypoxic regulation remains undefined. Thus, hypoxia promoter 6HRE-regulated plasmid VEGF $_{165}$ was constructed in this study and transfected into rat bone marrow-derived EPCs following in vitro isolation and cultivation to investigate the effect of $6 \mathrm{HRE}-\mathrm{VEGF}_{165}$ gene-modified EPCs on angiogenesis in ischemic myocardium and cardiac function following acute myocardial infarction (AMI). Furthermore, the differences in the effects on therapeutic angiogenesis in the ischemic myocardium and on the cardiac function of rats with AMI following transplantation of $6 \mathrm{HRE}-\mathrm{VEGF}_{165}$ gene-modified EPCs, $\mathrm{VEGF}_{165}$ gene-modified EPCs and normal EPCs were compared. No similar studies have been reported previously.

\section{Materials and methods}

Recombinant construction and identification of plasmids $p C M V-V E G F_{165}$ and $p 6 H R E-C M V-V E G F_{165}$. The single-copy HRE gene sequence from the pGEM-T vector (pGEM-T vector with HRE-CMV-mp gene; Shanghai Boya Corp., Shanghai, China) was connected to the 6-copy gene sequence using isocaudomers BamHI and BglII (Takara Corp., Tokyo, Japan) to synthesize a $6 \mathrm{HRE}-\mathrm{VEGF}_{165}$ gene expression plasmid (p6HRE-CMV-VEGF ${ }_{165}$-EGFP) and non-6HRE-regulated control plasmid (pCMV-VEGF ${ }_{165}$-EGFP) by molecular cloning techniques. The recombinant plasmids were then identified using restriction enzyme digestion, polymerase chain reaction (PCR) and DNA sequencing. The primers were as follows. 6HRE primer sequences: upstream, 5'-CGTACACGCCTA CCAGAT-3'; downstream, 5'-CCGCACTAGTGATTGGAT-3', 300 bp in length. VEGF $_{165}$ primer sequences: upstream, 5'-GCCACCATGAACTTTCTGCTG-3'; downstream, 5'-GGATCCTCACCGCCTCGGCTTGT-3', 630 bp in length.

Isolation, cultivation and identification of EPCs. EPCs were isolated from the femoral bone marrow of 80-100 g SPF male Sprague-Dawley rats (anesthetized with pentobarbital sodium, $30 \mathrm{mg} / \mathrm{kg}$ i.p.; provided by the Laboratory Animal Center of Chongqing Medical University). The cells were adjusted to a density of $2-4 \times 10^{6}$ cells/well and inoculated onto a 24 -well plate precoated with fibronectin (FN; Chemi-Con, Inc., Temecula, CA, USA) and cultured in M199 culture medium (HyClone Laboratories, Inc., Logan, UT, USA) containing 20\% FCS, $40 \mu \mathrm{g} / \mathrm{ml} \mathrm{BPE} \mathrm{(HyClone} \mathrm{Laboratories,} \mathrm{Inc.)} \mathrm{and} 1 \mathrm{ng} / \mathrm{ml}$ FGF-basic (PeproTech Inc., Rocky Hill, NJ, USA). The cells were incubated in a $37^{\circ} \mathrm{C}, 5 \% \mathrm{CO}_{2}$, humidity-saturated cell culture incubator (Forma Scientific, Inc., Marietta, OH, USA). The culture medium was exchanged after 3 days of cultivation for the first time, and the medium was then exchanged every 2 days to observe the morphological changes of the cells. At days 5 and 11 of cultivation, cells were obtained for the detection of the specific cell surface antigens CD133 (Abcam, Inc., Cambridge, MA, USA), CD34 (Millipore Corp., Billerica, MA, USA) and the VEGF receptor (VEGFR; Millipore
Corp.), and the phagocytosis of Dil-ac-LDL (Invitrogen Corp., Carlsbad, CA, USA) by the cells using immunofluorescent cytochemical staining (Leica, Mannheim, Germany). The rat experiments were approved by the local institutional animal research committee [SCXK (Yu) 2007-0001].

The successfully cultured EPCs were allocated to one of three groups: EPCs transfected with the recombinant plasmid p6HRE-CMV-VEGF ${ }_{165}$-EGFP (group HV), EPCs transfected with the recombinant plasmid pCMV-VEGF $_{165}$-EGFP (group V) and control EPCs transfected with the plasmid pCMV-MCS-EGFP (group E, EPCs control group). The gene transfection with plasmids $\mathrm{pCMV-VEGF}{ }_{165}$ and p6HRE-CMVVEGF $_{165}$ was conducted using liposome Lipofectamine 2000 (Invitrogen Corp.); $24 \mathrm{~h}$ after gene transfection, the positive rate of transfection was calculated by flow cytometry (BectonDickinson Co., Franklin Lakes, NJ, USA) as follows: number of positive cells carrying fluorescence/total number of detected cells x $100 \%$.

Dil-ac-LDL-labeled EPCs. EPCs cultured for 10 days were added to Dil-ac-LDL $(10 \mu \mathrm{g} / \mathrm{ml})$ and incubated for $5 \mathrm{~h}$. The culture medium was then exchanged and the cells were observed under an inverted fluorescence microscope to determine the ratio of fluorescently labeled cells. Successfully labeled EPCs were digested with $0.25 \%$ trypsin, centrifuged, resuspended in PBS and adjusted to a density of $2 \times 10^{7}$ cells $/ \mathrm{ml}$. The EPC suspension $(0.5 \mathrm{ml})$ was injected via the tail vein into rats with a successfully established model of AMI. Three days after cell transplantation, the rats were sacrificed. Heart specimens were removed from the rats, rinsed with PBS and cut into frozen sections $(10 \mu \mathrm{m})$ to enable observation of the location of the fluorescence-labeled cells in the cardiac sections under an inverted microscope.

Establishment of the rat model with AMI and cell transplantation. MI was induced in adult male Sprague-Dawley rats weighing 180-230 g (anesthetized with pentobarbital sodium, $30 \mathrm{mg} / \mathrm{kg}$ i.p.; provided by the Laboratory Animal Center of Chongqing Medical University) by permanent ligation of the left anterior descending coronary artery (8). The animal study was approved by the Chongqing Medical University ethics review board [SCXK (Yu) 2007-0001]. The rats with a successfully established model of AMI were divided into 4 groups (each $n=10$ ) and injected via the tail vein with Dil-ac-LDL fluorescence-labeled p6HRE-CMV-VEGF 165 -transfected EPCs $\left(2 \times 10^{7}\right.$ cells $\left./ \mathrm{ml}\right)$, pCMV-VEGF $165^{-}$transfected EPCs $\left(2 \times 10^{7} \mathrm{cells} / \mathrm{ml}\right), \mathrm{EPCs}\left(2 \times 10^{7} \mathrm{cell} / \mathrm{s} / \mathrm{ml}\right)$ or $0.5 \mathrm{ml}$ normal saline. The sham surgery group was regarded as the control $(n=10)$. One week later, 4 rats (one randomly selected from each group) were sacrificed by cervical dislocation and their hearts were rapidly removed. The myocardial tissues near the left ventricular infarction were excised, rinsed with pre-cooled normal saline and DEPC, and equally divided into 2 samples for reverse transcription-PCR (RT-PCR) and western blot detection of the $\mathrm{VEGF}_{165}$ gene and protein expression levels. At week 4, cardiac echocardiography was performed on the remaining rats to evaluate cardiac function and cardiac tissues. The sacrificed rats were rinsed with normal saline and fixed in $4 \%$ paraformaldehyde in preparation for further use. 
Detection of VEGF 165 gene expression under hypoxia promoter regulation. EPCs successfully transfected with p6HRE-CMV-VEGF ${ }_{165}$ and pCMV-VEGF $_{165}$ were placed in an $\mathrm{N}_{2}$ incubator containing $1 \% \mathrm{O}_{2} 36 \mathrm{~h}$ after transfection. Following 12-h hypoxic incubation, the EPCs were removed from the incubator and allocated to either a transfection control group or a normal oxygen concentration incubation control group. The levels of $\mathrm{VEGF}_{165} \mathrm{mRNA}$ and protein expression were detected using RT-PCR and western blotting, respectively. The levels of $\mathrm{VEGF}_{165}$ mRNA and protein expression in the ischemic myocardium 1 week after cell transplantation were also detected using the same assays.

The mRNA in the EPCs and the ischemic myocardium was extracted and reversely transcribed into cDNA with reference to the total DNA extracted standard protocol. The absorbance ratio between $\mathrm{VEGF}_{165}$ and $\beta$-actin ( $\beta$-actin upstream primer, 5'-CCCATCTATGAGGGTTACGC-3'; downstream primer, 5'-TTTAATGTCACGCACGATTTC-3', 150 bp in length) was calculated following analysis using the software Quantity One 4.4.0 and represented the relative expression level.

Total protein was extracted from the EPCs and the ischemic myocardium with reference to the protein extracted standard protocol; $20 \mu \mathrm{l}$ supernatant was obtained to determine the protein concentration using bicinchoninic acid, the wavelength was measured using a microplate reader and the protein concentration was calculated from the standard curve. The VEGF $_{165}$ protein was isolated using $120 \mathrm{ml} / 1$ SDS-PAGE gel electrophoresis, electrotransferred onto a PVDF membrane and blocked with $50 \mathrm{~g} / 1$ evaporated skimmed milk. After 1 -h incubation at room temperature, the blocking buffer was removed and the protein was combined with rabbit anti-rat VEGF primary antibody (Millipore Corp., 1:500). The protein was then added to secondary antibody anti-rabbit immunoglobulin IgG (1:1000, Zhongshan Jin Qiao Biotechnology Co., Ltd., Beijing, China). Enhanced chemiluminescence was applied for color development and the grayscale of the band was analyzed using a gel image analysis system.

Hematoxylin and eosin $(H \& E)$ and immunohistochemical staining of myocardial tissues. Infarct size (IS) was determined by H\&E staining. In brief, sections of the left ventricle were immersed in fixative solution, dehydrated and then embedded in paraffin. Afterwards, 5-mm-thick histological slices were obtained and stained with H\&E. The endocardial and epicardial circumferences of the infarcted tissue and the left ventricle were determined using image analysis software (Image-Pro Plus 4.5). The IS was calculated as (endocardial + epicardial circumference of the infarcted tissue)/(endocardial + epicardial circumference of the left ventricle) and expressed as a percentage. The capillary density of the heart was detected by immunohistochemical staining using a 2-Step Immunohistochemistry Detection kit (Zhongshan Golden Bridge Biotechnology Co., Ltd., Beijing, China). Specific procedures in line with the operation manual were followed. The working concentration of rabbit anti-rat VIII primary antibodies was 1:50. The primary antibodies were replaced by PBS buffer as the negative control.

Statistical processing. All measurement data were expressed as the mean \pm SD and statistically analyzed using SPSS 13.0 statistical software. The means of mRNA and protein expression levels among different groups were compared by 1-way analysis of variance (ANOVA) and multiple comparisons between each pair of groups were conducted using the 2-sided Tukey's test. $\mathrm{P}<0.05$ was considered to indicate a statistically significant result.

\section{Results}

Identification results for in vitro cultivation of EPCs. EPCs isolated and cultured in vitro adhered to the plate after 3 days of cultivation and displayed a radial and proliferative growing state 7 days later. Immunofluorescent cytochemical staining revealed that the EPC surface antigens CD34 and CD133 stained positive on day 5, but the expression of CD34 was more intense than that of CD133 (Fig. 1). By day 11, EPC surface antigen CD133 was essentially unexpressed, but CD34 (green) and VEGFR (red) were stained positive. In addition, the cells phagocytized Dil-ac-LDL and the positive cells displayed red fluorescence under the inverted microscope (Fig. 2). Flow cytometry carried out $24 \mathrm{~h}$ later detected EPCs successfully cultured using cationic liposomes and revealed the expression of green fluorescence protein by $40 \%$ of the EPCs following transfection.

\section{Detection of $V E G F_{165}$ gene expression}

Detection of VEGF ${ }_{165} m R N A$ and protein expression under in vitro regulation of the hypoxia promoter. The levels of VEGF $_{165}$ mRNA and protein expression in the EPCs transfected with the recombinant plasmid p6HRE-CMV-VEGF 165 were significantly higher than those in the EPCs transfected with the recombinant plasmid pCMV-VEGF ${ }_{165}$ and those not transfected with any recombinant plasmid $(\mathrm{P}<0.05)$ under hypoxic induction (Fig. 3). Nonetheless, the levels of $\mathrm{VEGF}_{165}$ gene expression in the p6HRE-CMV-VEGF ${ }_{165}$-transfected and untransfected EPCs were comparatively equivalent but significantly lower than those in the EPCs transfected with pCMV-VEGF $_{165}$ (Fig. 3) when cultivated in normal oxygen conditions. The level of $\mathrm{VEGF}_{165}$ gene expression by the pCMV-VEGF 165 -transfected EPCs appeared higher under hypoxic conditions than in normal oxygen conditions, but the difference was not statistically significant ( $P>0.05$; Fig. 3).

Detection results of $V E G F_{165}$ gene expression under in vivo regulation of the hypoxia promoter. The present results revealed that the levels of VEGF $_{165}$ mRNA and protein expression in the EPCs transplanted near the infarction area of the rats were significantly higher in the p6HRE-CMV-VEGF ${ }_{165}$-transfected EPCs than those in the other groups $(\mathrm{P}<0.05)$, and that $\mathrm{VEGF}_{165}$ gene expression levels in the normal EPC transplantation group were higher than those in the AMI group $(\mathrm{P}<0.05)$ but lower than those in the pCMV-VEGF ${ }_{165}$-transfected EPC transplant group ( $\mathrm{P}<0.05$; Fig. 4$)$.

$H \& E$ staining and immunohistochemical results of the myocardium near the infarction area. Rats of different experimental groups were sacrificed at week 4 after cell transplantation. Their cardiac tissues were removed, embedded in paraffin and cut into sections. H\&E staining revealed that the myocardium of normal cardiac tissues was arranged in order without manifestations of muscle bundle fracture or fiber 

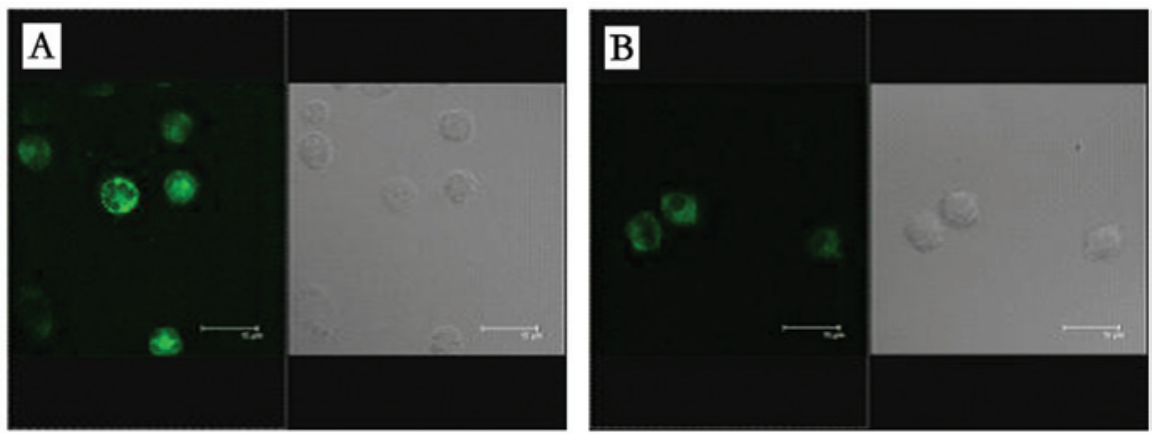

Figure 1. Expression of endothelial progenitor cell (EPC) surface antigens on day 5. (A) CD34+ and (B) CD133+ EPCs (laser confocal microscope, x2000).
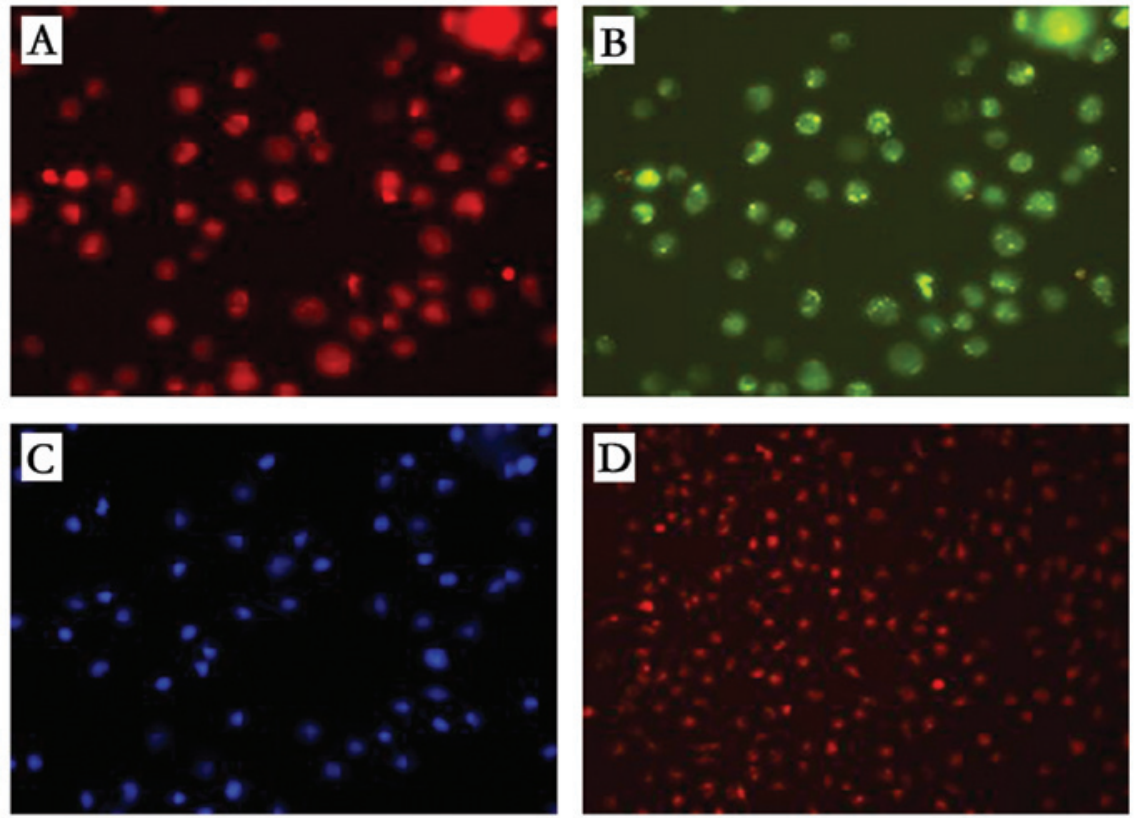

Figure 2. Expression of endothelial progenitor cell (EPC) surface antigens on day 11. (A) EPC surface antigen vascular endothelial growth factor receptor (VEGFR ; red, upright fluorescence microscope, x20); (B) EPC surface antigen CD34 (green, upright fluorescence microscope, x20); (C) cell nuclei of EPCs (blue, upright fluorescence microscope, x20); and (D) EPCs stained with Dil-ac-LDL (red, inverted fluorescence microscope, x10).

hyperplasia, but that myocardial disarrangement and muscle bundle fracture was observed following myocardial infarction with the proliferation of a large number of fiber cells in the disarranged myocardium (data not shown).

The infarct area size in the $\mathrm{pCMV} \mathrm{VEGF}_{165}$-transfected EPC transplantation group was smaller than those in the untransfected EPC transplantation and AMI groups but larger than that in the p6HRE-CMV-VEGF ${ }_{165}$-transfected EPC transplantation group by $\mathrm{H} \& \mathrm{E}$ staining $(\mathrm{P}<0.05)$. The infarct area size in the untransfected EPC transplantation group was not observed to be significantly different from that in the AMI group ( $\mathrm{P}>0.05$; Fig. 6A).

The results of capillary area calculation following the factor VIII staining of the vascular endothelial cells in the myocardium near the infarction area into brown or brownish yellow particles using the SABC immunocytochemical detection kit are presented in Fig. 5. The capillary density determination indicated that the numbers of $\mathrm{VIII}^{+}$vessels in the pCMV-VEGF ${ }_{165^{-}}$and p6HRE-CMV-VEGF ${ }_{165}$-transfected EPC transplantation groups were higher than those in the sham surgery and AMI groups 4 weeks post-MI; those in the p6HRE-CMV-VEGF ${ }_{165}$-transfected EPC group were significantly higher $(\mathrm{P}<0.05)$. The capillary density in the normal EPC transplantation group was lower than that in the sham group $(\mathrm{P}<0.05)$ and not significantly different from that in the AMI group $(\mathrm{P}>0.05)$.

Results of cardiac function testing of the rats by echocardiography. Evalution of the cardiac functions of the rats by echocardiography at week 4 following cell transplantation revealed the formation of false ventricular aneurysms on the left ventricular wall in the infarction group. The rats in the p6HRE-CMV-VEGF ${ }_{165}$-transfected EPC transplantation group had a significantly lower left ventricular end-diastolic diameter but higher ejection fraction than did the other experimental groups (Fig. 6; $\mathrm{P}<0.05$ ). 

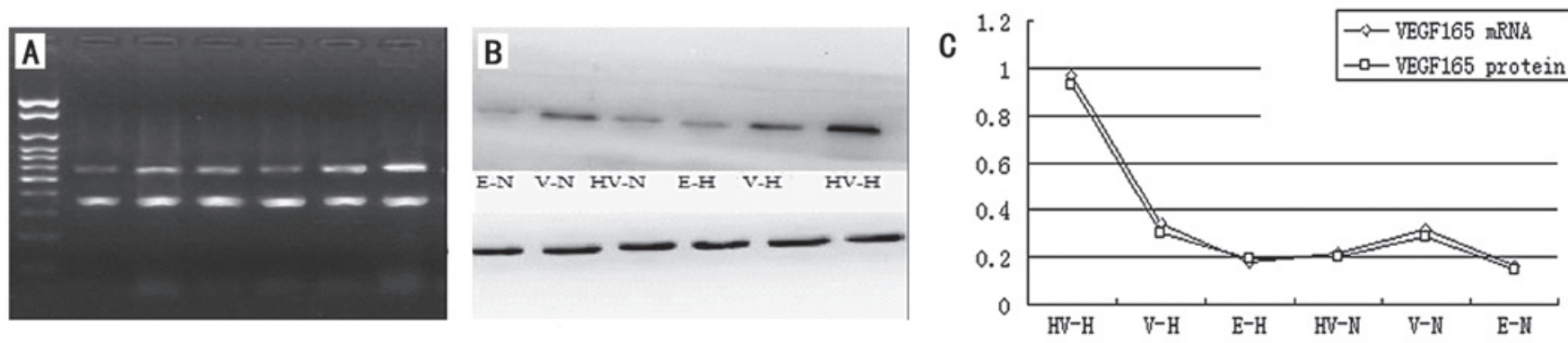

Figure 3. Vascular endothelial growth factor 165 (VEGF $\left.{ }_{165}\right)$ mRNA and protein expression by gene-modified endothelial progenitor cells (EPCs). (A) Reverse transcription-polymerase chain reaction (RT-PCR) detection of VEGF $_{165}$ mRNA expression; (B) western blot detection of VEGF $_{165}$ protein expression; (C) relative expression levels of $\mathrm{VEGF}_{165} \mathrm{mRNA}$ and protein in gene-modified EPCs after 12-h induction under hypoxic and normal oxygen cultivation conditions. HV, EPCs transfected with the recombinant plasmid p6HRE-CMV-VEGF ${ }_{165}$; V, EPCs transfected with the recombinant plasmid pCMV-VEGF ${ }_{165}$; E, EPCs; H, hypoxic cultivation conditions; $\mathrm{N}$, normal oxygen cultivation conditions.
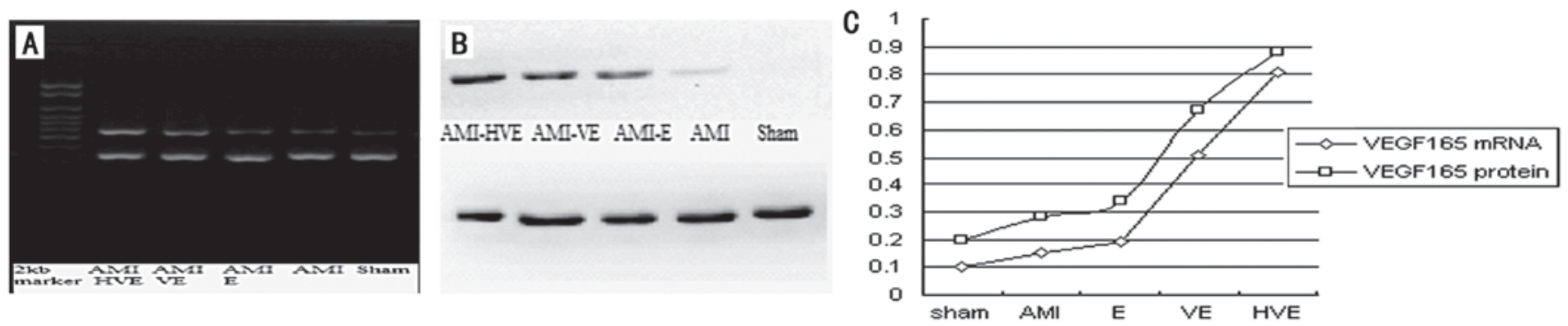

Figure 4. Expression of vascular endothelial growth factor $165\left(\mathrm{VEGF}_{165}\right)$ mRNA and protein in the ischemic myocardium. (A) Reverse transcription-polymerase chain reaction (RT-PCR) detection of $\mathrm{VEGF}_{165} \mathrm{mRNA}$ expression; (B) western blot detection of $\mathrm{VEGF}_{165}$ protein expression; (C) relative expression levels of $\mathrm{VEGF}_{165} \mathrm{mRNA}$ and protein in the ischemic myocardium 1 week after acute myocardial infarction (AMI) in rats. Sham, sham surgery group; E, endothelial progenitor cell (EPC) transplantation group; VE, pCMV-VEGF ${ }_{165}$-transfected EPC transplantation group; HVE, p6HRE-CMV-VEGF ${ }_{165}$-transfected EPC transplantation group.

\section{Discussion}

The present study found that: i) hypoxia-specific expression of the $\mathrm{VEGF}_{165}$ gene carried by EPCs and regulated by $6 \mathrm{HRE}$ could be achieved in vivo and in vitro; and ii) when the transplantation of EPCs was modified by the $\mathrm{VEGF}_{165}$ gene and regulated by $6 \mathrm{HRE}$, it produced a stronger therapeutic effect in promoting angiogenesis. It radically improved cardiac function following myocardial infarction, reducing the myocardial infarct size.

The VEGF gene is the most classic angiogenic growth factor that has been demonstrated to have an effect on angiogenesis by basic and clinical studies. In combination with VEGFR-1 (9), it promotes the vascular regeneration of ischemic limbs, heart and brain (10-12), and increases the collateral circulation and improves the function of ischemic tissue. Studies have shown that its overexpression and expression in non-target organs may cause inflammation, edema, the formation of vascular tumors and other side effects, and may even trigger other grave consequences. Nevertheless, the primary focus of the present study was to ensure safe VEGF gene transfer. This is likely to be effective in the treatment of ischemic diseases. The present study primarily focused on determining the specificity of VEGF gene expression regulated by hypoxia.

Previous studies have identified a short gene sequence upstream of the VEGF gene promoter, namely the HRE, which binds with hypoxia inducible factor-1 (HIF-1) specifically under hypoxic conditions. This initiates the translation and expression of target genes, indicating that hypoxia is a natural VEGF gene-regulating factor. However, studies by Tang and other researchers have found no upregulatory role for the single-copy HRE promoter under hypoxic conditions, whereas subsequent studies have confirmed that series of multi-copy HRE promoters are able to enhance the transcriptional activity of the target gene under hypoxic conditions (13). The regulation capacity of HRE is proportional to the copy number (14), while there is no expression of target genes under normal oxygen concentrations (15). Thus, multiple copies of HRE induce a switch effect in regulating target genes in hypoxic conditions (16), enhancing the intensity of target gene expression and specificity for hypoxia.

$\mathrm{Su}$ et al (17) have regulated VEGF with MLC-2 and 9HRE, and identified that the gene expression level of VEGF in the regulated myocytes after $16 \mathrm{~h}$ of hypoxia inducement in vitro was approximately twice that in the control group and approximately 8-fold higher than that in the non-hypoxic group. However, there was almost no expression of the target gene in the regulated group under non-hypoxic conditions. It was also significantly lower than that in the control group. A gene expression study using 2 regulators (9HRE and ODD), as reported by Fomicheva et al (18), revealed that the expression levels of target genes increased 1,000-fold when induced by $0.5 \%$ oxygen after $48 \mathrm{~min}$. Recently, Wei et al reported the 

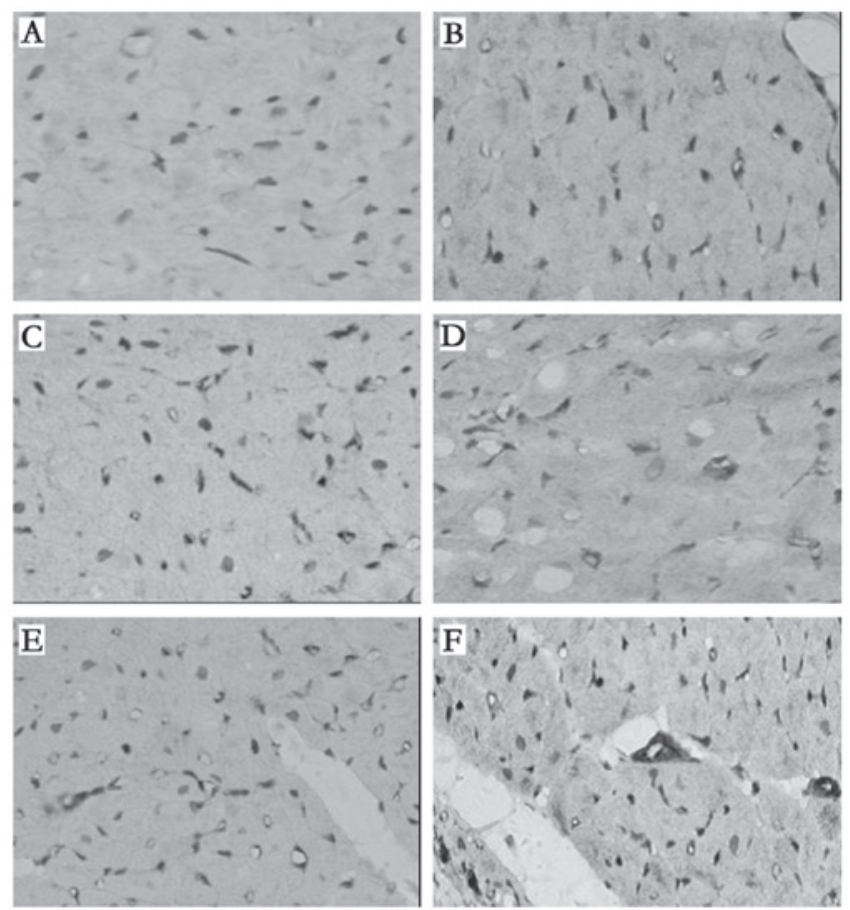

Figure 5. Factor VIII staining of vascular endothelial cells in the myocardium. Factor VIII staining for (A) negative control, (B) sham surgery, (C) acute myocardial infarction (AMI), (D) epithelial progenitor cell (EPC) transplantation, (E) pCMV-VEGF 165 -transfected EPC transplantation (VE) and (F) p6HRE-CMV-VEGF ${ }_{165}$-transfected EPC transplantation (HVE) groups (upright microscope, $\mathrm{x} 40$ ).

construction of a CD151 gene vector regulated by HRE using an adeno-associated virus which was transfected into an ischemic heart. The results revealed enhanced VEGF gene expression of CD151 and vascularization in the ischemic myocardium (19).

In the current study, the $\mathrm{VEGF}_{165}$ gene expression plasmid regulated by $6 \mathrm{HRE}$ was constructed and transfected into EPCs cultured in vitro. These were then placed in a $1 \% \mathrm{O}_{2}$ hypoxic incubator. After 12-h incubation, the $\mathrm{VEGF}_{165}$ mRNA and protein expression levels were approximately 4-fold higher than those in the normoxic control group and were approximately 3 -fold higher than those in the group without $6 \mathrm{HRE}$ regulation under hypoxic conditions. However, VEGF mRNA and protein expression were at low levels in the 6HRE-regulated group cultured in normoxic conditions. Thereafter, gene transfected EPCs were transplanted into the myocardium and the expression level of VEGF in the 6HRE-regulated group was determined to be significantly greater than that in the non-HRE group. This further indicated that $6 \mathrm{HRE}$ exerted a switch effect in regulating the expression of the $\mathrm{VEGF}_{165}$ gene under hypoxic conditions and may improve the angiogenic effect of $\mathrm{VEGF}_{165}$ gene therapy, enhancing the targeting of anoxic tissues with gene therapy.

EPCs with the remedial angiogenesis gene have improved targeting, transportation and therapeutic effects. EPCs are able to specifically heal damaged vessels, differentiate endothelial cells, secrete various angiogenic growth factors, and promote angiogenesis and neovascularization. It has been widely verified that they have a role in therapeutic angiogenesis and are without the potential risk of arrhythmia promotion $(19,20)$.
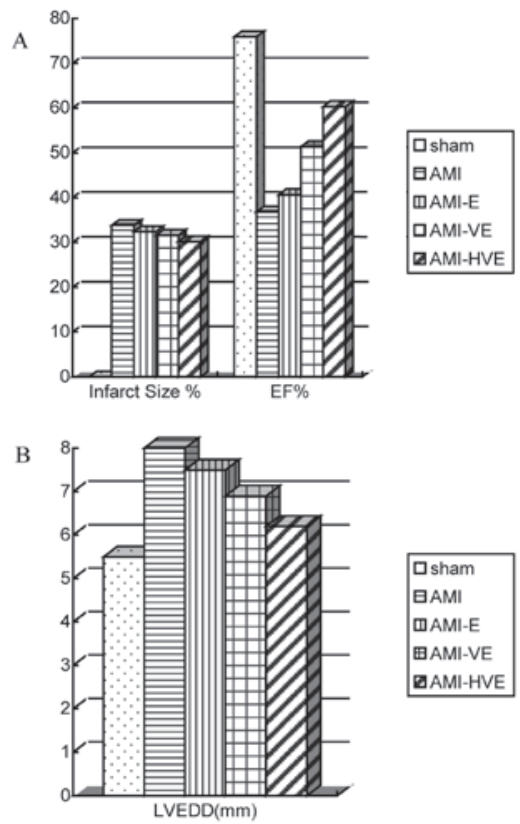

Figure 6. Detection results of cardiac function indices of rats in different groups at week 4. Sham, sham surgery group; AMI, acute myocardial infarction group; E, endothelial progenitor cell (EPC) transplantation group; VE, pCMV-VEGF ${ }_{165}$-transfected EPC transplantation group; HVE, p6HRE-CMV-VEGF ${ }_{165}$-transfected EPC transplantation group.

A study conducted by Iwaguro et al involving the transplantion of VEGF gene-modified EPCs identified for the first time that the ability of EPCs to promote angiogenesis was enhanced in the ischemic extremities of animals (21). Since then, a number of studies have found that the transplantation of gene-modified EPCs promotes restoration of the bloodstream in ischemic extremities, improves the function of ischemic tissue and reduces the frequency of EPC transplantation $(21,22)$. In addition, it increases the number of EPCs in local ischemic tissue (23), enhancing the migration of EPCs, tubular formation and survival. It also relieves the extent of myocardial perfusion deficiency (24). Hence, gene-modified EPCs have a strong angiogenetic effect, with reduced ectopic expression of genes. It is not yet clear whether EPCs modified by the $\mathrm{VEGF}_{165}$ gene under the regulation of hypoxia play an important role in increasing angiogenesis, improving cardiac function following AMI and reducing myocardial infarct size.

This study identified that capillary density was significantly higher in the $6 \mathrm{HRE}-\mathrm{VEGF}_{165}$ gene-modified EPC transplantation group than in the non-6HRE-VEGF ${ }_{165}$ gene-modified EPC transplantation, normal EPC transplantation and normal saline control groups. This significant contrast in capillary density was observed at the ischemic myocardium near the myocardial infarction area at week 4 . This was in accordance with the aforementioned results. Investigation of the cardiac function of the rats by echocardiography revealed that the left ventricular end diastolic volume and myocardial infarction areas in the $6 \mathrm{HRE}-\mathrm{VEGF}_{165}$ gene-modified EPC transplantation group were significantly reduced compared with those in the other three groups. However, the left ventricular ejection fraction was significantly higher than that in the other three groups. In the $6 \mathrm{HRE}-\mathrm{VEGF}_{165}$ gene-modified EPC transplantation group, due to the homing of EPCs to the highly ischemic and hypoxic 
ventricle near the infarction area, the hypoxia promoter rapidly and powerfully initiated and induced the expression of $\mathrm{VEGF}_{165}$ mRNA and protein. It increased the regional and circulating amount of VEGF, further promoting the release, homing and differentiation of EPCs in the rats and also intensified the secretion of regional growth factors (23), speeding up the formation and maturation of new blood vessels, rescuing the dying myocardium and reducing the area of myocardial infarction and deficiency in myocardial perfusion $(23,24)$. This ameliorated the cardiac function of the rats compared with that in the non-6HRE-VEGF ${ }_{165}$ gene-modified EPC transplantation group. Dong et al (25) reported that following transfection of the AAV-9HRE-VEGF virus into ischemic myocardium, the 9HRE-VEGF gene was able to further ameliorate the function of ischemic rat hearts and upregulate the level of CD31 cells, supporting the speculation in the current study. 6HRE hypoxia regulation, the angiogenic growth factor gene $\left(\mathrm{VEGF}_{165}\right)$ and progenitor cell transplantation were effectively combined in the present experiment. The results verified that the outcome of therapeutic angiogenesis and heart function improved with the combination of these three methods. It was superior to single EPC transplantation and EPC transplantation combined with the VEGF gene.

In conclusion, the results of this study confirmed that the transplantation of $\mathrm{VEGF}_{165}$ gene-modified EPCs under $6 \mathrm{HRE}$ regulation significantly reduced the left ventricular end diastolic volume of rats. It also significantly increased the left ventricular ejection fraction and capillary density in the ischemic zone. Therefore, a method that combines the expression of the $6 \mathrm{HRE}$ hypoxia regulation gene with progenitor cell transplantation is safe and effective for the treatment of ischemic heart diseases and other ischemic diseases.

\section{Acknowledgements}

The authors wish to thank Yang Wang, PhD for offering important suggestions regarding this manuscript. This study was supported by the National Natural Science Foundation of China (81100088/H0203), the Important Project of Chongqing Health Administration (20090113) and the Chongqing Medical University Science Foundation.

\section{References}

1. Korpisalo P, Rissanen TT, Bengtsson T, et al: Therapeutic angiogenesis with placental growth factor improves exercise tolerance of ischaemic rabbit hindlimbs. Cardiovasc Res 80: 263-270, 2008.

2. Leong-Poi H, Kuliszewski MA, Lekas M, et al: Therapeutic arteriogenesis by ultrasound-mediated VEGF165 plasmid gene delivery to chronically ischemic skeletal muscle. Circ Res 101: 295-303, 2007.

3. Lähteenvuo JE, Lähteenvuo MT, Kivelä A, et al: Vascular endothelial growth factor-B induces myocardium-specific angiogenesis and arteriogenesis via vascular endothelial growth factor receptor-1 and neuropilin receptor-1-dependent mechanisms. Circulation 119: 845-856, 2009.

4. Fortuin FD, Vale P,Losordo DW, et al: One-year follow-up of direct myocardial gene transfer of vascular endothelial growth factor-2 using naked plasmid deoxyribonucleic acid by way of thoracotomy in no-option patients. Am J Cardiol 92: 436-439, 2003.

5. Losordo DW, Vale PR, Hendel RC, et al: Phase 1/2 placebo-controlled, double-blind, dose-escalating trial of myocardial vascular endothelial growth factor 2 gene transfer by catheter delivery in patients with chronic myocardial ischemia. Circulation 105: 2012-2018, 2002.
6. Sato K, Wu T, Laham RJ, et al: Efficacy of intracoronary or intravenous VEGF165 in a pig model of chronic myocardial ischemia. J Am Coll Cardiol 37: 616-623, 2001.

7. Zemani F, Silvestre JS, Fauvel-Lafeve F, et al: Ex vivo priming of endothelial progenitor cells with SDF-1 before transplantation could increase their proangiogenic potential. Arterioscler Thromb Vasc Biol 28: 644-650, 2008.

8. Xiao J, She Q, Wang Y, et al: Effect of allopurinol on cardiomyocyte apoptosis in rats after myocardial infarction. Eur J Heart Fail 11: 20-27, 2009.

9. Clayton JA, Chalothorn D and Faber JE: Vascular endothelial growth factor-A specifies formation of native collaterals and regulates collateral growth in ischemia. Cir Res 103: 1027-1036, 2008.

10. Xie D, Li Y, Reed EA, et al: An engineered vascular endothelial growth factor-activating transcription factor induces therapeutic angiogenesis in ApoE knockout mice with hindlimb ischemia. J Vasc Surg 44: 166-175, 2006.

11. Toyota E, Warltier DC, Brock T, et al: Vascular endothelial growth factor is required for coronary collateral growth in the rat. Circulation 112: 2108-2113, 2005.

12. Sun Y, Jin K, Xie L, et al: VEGF-induced neuroprotection, neurogenesis, and angiogenesis after focal cerebral ischemia. J Clin Invest 111: 1843-1851, 2003.

13. Tang Y: Gene therapy for myocardial ischemia using the hypoxia-inducible double plasmid system. Methods Mol Med 112: 37-47, 2005.

14. Harada H, Kizaka-Kondoh S, Itasaka S, Shibuya K, et al: The combination of hypoxia-response enhancers and an oxygen-dependent proteolytic motif enables real-time imaging of absolute HIF-1 activity in tumor xenografts. Biochem Biophys Res Commum 360: 791-796, 2007.

15. Dulak J, Zagorska A, Wegiel B, et al: New strategies for cardiovascular gene therapy: regulatable pre-emptive expression of pro-angiogenic and antioxidant genes. Cell Biochem Biophys 44: 31-42, 2006.

16. Su H and Kan YW: Adeno-associated viral vector-delivered hypoxia-inducible gene expression in ischemic hearts. Methods Mol Biol 366: 331-342, 2007.

17. Su H, Joho S, Huang Y, et al: Adeno-associated viral vector delivers cardiac-specific and hypoxia-inducible VEGF expression in ischemic mouse hearts. Proc Natl Acad Sci USA 101: 16280-16285, 2004.

18. Fomicheva EV, Turner II, Edwards TG, et al: Double oxygen-sensing vector system for robust hypoxia/ischemiaregulated gene induction in cardiac muscle in vitro and in vivo. Mol Ther 16: 1594-1601, 2008.

19. Wei Q, Huang XL, Lin JY, et al: Adeno associated viral vector-delivered and hypoxia response element-regulated CD151 expression in ischemic rat heart. Acta Pharmacol Sin 32: 201-208, 2011.

20. Losordo DW, Schatz RA, White CJ, et al: Intramyocardial transplantation of autologous CD34 ${ }^{+}$stem cells for intractable angina: a phase I/IIa double-blind, randomized controlled trial. Circulation 115: 3165-3172, 2007.

21. Iwaguro H, Yamaguchi J, Kalka C, et al: Endothelial progenitor cell vascular endothelial growth factor gene transfer for vascular regeneration. Circulation 105: 732-738, 2002.

22. Murasawa S, Llevadot J, Silver M, et al: Constitutive human telomerase reverse transcriptase expression enhances regenerative properties of endothelial progenitor cells. Circulation 106: 1133-1139, 2002

23. Yu JX, Huang XF, Lv WM, et al: Combination of stromal-derived factor-1alpha and vascular endothelial growth factor gene-modified endothelial progenitor cells is more effective for ischemic neovascularization. J Vasc Surg 50: 608-616, 2009.

24. Chen SY, Wang F, Yan XY, et al: Autologous transplantation of EPCs encoding FGF1 gene promotes neovascularization in porcine model of chronic myocardial ischemia. Int J Cardiol 135: 223-232, 2009.

25. Dong HY, Wang Q, Zhang Y, et al: Angiogenesis induced by hVEGF165 gene controlled by hypoxic response elements in rabbit ischemic myocardium. Exp Biol Med (Maywood) 234: 1417-1424, 2009. 